\begin{tabular}{|r|l|}
\hline \multicolumn{2}{|c|}{ Statistica Sinica Preprint No: SS-2021-0070 } \\
\hline Title & $\begin{array}{l}\text { Semiparametric Estimation of Probabilistic Index } \\
\text { Models: Efficiency and Bias }\end{array}$ \\
\hline Uanuscript ID & SS-2021-0070 \\
\hline DRL & http://www.stat.sinica.edu.tw/statistica/ \\
\hline Complete List of Authors & $\begin{array}{l}\text { Karel Vermeulen, } \\
\text { Jan De Neve, } \\
\text { Gustavo Amorim, } \\
\text { Olivier Thas and } \\
\text { Stijn Vansteelandt }\end{array}$ \\
\hline Corresponding Author & Jan De Neve \\
\hline E-mail & Jan.DeNeve@UGent.be \\
\hline Notice: Accepted version subject to English editing. \\
\hline
\end{tabular}


Statistica Sinica

\title{
SEMIPARAMETRIC ESTIMATION OF PROBABILISTIC INDEX MODELS: EFFICIENCY AND BIAS
}

\author{
Karel Vermeulen, Jan De Neve, Gustavo Amorim, \\ Olivier Thas and Stijn Vansteelandt \\ Ghent University, Vanderbilt University, \\ Hasselt University, London School of Hygiene and Tropical Medicine
}

\begin{abstract}
Many well known rank tests can be viewed as score tests under probabilistic index models: regression models for the conditional probability that the outcome of one randomly chosen subject exceeds the outcome of another independently chosen subject. Probabilistic index models (PIMs) provide a natural regression framework for nonparametric rank tests. In addition, PIMs supplement these tests with effect sizes and ease the development of more flexible tests e.g., tests which allow for covariate adjustment. Inference for probabilistic index models is currently based on an estimator, referred to as the standard estimator, that was heuristically derived. By appealing to semiparametric theory and a Hoeffding decomposition, we rigorously derive the class of all consistent and asymptotically normal estimators for the parameters indexing a probabilistic in-
\end{abstract}


dex model. We identify the (locally) semiparametric efficient estimator in this class and we derive a second estimator which has smaller second-order finitesample bias. The properties of the estimators are theoretically and empirically evaluated. The heuristic standard estimator turns out to be the preferred estimator in practice as it is computationally superior to both the efficient and the bias-reduced estimator, and only suffers from a minor loss in efficiency. We propose a partition strategy to further improve the computational performance of the standard estimator.

Key words and phrases: Cross correlation, Influence function, Second-order bias, Semiparametric estimation, U-process.

\section{Introduction}

Probabilistic index models (PIMs, Thas et al., 2012) form a class of semiparametric models for the probability that the outcome of one randomly chosen subject exceeds the outcome of another independently chosen subject, as a function of covariates. Let $\left\{\mathbf{Z}_{i}^{T}=\left(Y_{i}, \mathbf{X}_{i}^{T}\right): i=1, \ldots, n\right\}$ denote a sample of $n$ independent and identically distributed (IID) random vectors, where $Y_{i}$ denotes the outcome of interest associated with the $p$-dimensional vector of covariates $\mathbf{X}_{i}$. A PIM is defined by the constraint

$$
\mathrm{P}\left(Y_{i} \preccurlyeq Y_{j} \mid \mathbf{X}_{i}, \mathbf{X}_{j}\right)=m\left(\mathbf{X}_{i}, \mathbf{X}_{j} ; \boldsymbol{\beta}_{0}\right)
$$


with $\mathrm{P}\left(Y_{i} \preccurlyeq Y_{j} \mid \mathbf{X}_{i}, \mathbf{X}_{j}\right):=\mathrm{P}\left(Y_{i}<Y_{j} \mid \mathbf{X}_{i}, \mathbf{X}_{j}\right)+0.5 \mathrm{P}\left(Y_{i}=Y_{j} \mid \mathbf{X}_{i}, \mathbf{X}_{j}\right)$. This probability is referred to as the probabilistic index (PI). The function $m(\cdot)$ is a known function with range $[0,1]$, smooth in the $p$-dimensional parameter vector $\boldsymbol{\beta}$ and satisfying the antisymmetry condition $m\left(\mathbf{X}_{i}, \mathbf{X}_{j} ; \boldsymbol{\beta}\right)=$ $1-m\left(\mathbf{X}_{j}, \mathbf{X}_{i} ; \boldsymbol{\beta}\right)$. The function $m(\cdot)$ typically takes the form $m\left(\mathbf{X}_{i}, \mathbf{X}_{j} ; \boldsymbol{\beta}\right)=$ $g^{-1}\left\{\left(\mathbf{X}_{j}-\mathbf{X}_{i}\right)^{T} \boldsymbol{\beta}\right\}$, with $g(\cdot)$ an appropriate link-function, such as the probit- or logit-link. We let $\boldsymbol{\beta}_{0}$ denote the true but unknown value of $\boldsymbol{\beta}$ that corresponds with the observed data law.

Thas et al. (2012) demonstrate that PIMs form a versatile class of models applicable to continuous and ordinal outcomes and they establish connections with the Cox proportional hazards model and rank regression. De Neve and Thas (2015) illustrate how PIMs provide a unified regression framework for many rank tests such as the Wilcoxon-Mann-Whitney (WMW), Kruskal-Wallis and Friedman rank tests. An attractive feature of a PIM is that, in addition to hypothesis testing, it enables estimating effect sizes with an informative interpretation; however, we refer to the illustration in Section 4 for a cautionary remark on the interpretation of a PI. De Neve and Thas (2015) also illustrate how PIMs can be used to construct new rank tests for complicated designs. This is further convincingly demonstrated in Vermeulen et al. (2015) who employ PIMs to increase the 
power of the WMW test by including auxiliary covariate information in randomised designs.

The parameter estimation and statistical inference proposed in Thas et al. (2012) relies on reformulating the PIM (1.1) as a semiparametric conditional moment model (Chamberlain, 1987, Newey, 1988) for the pseudoobservations $\mathrm{I}\left(Y_{i} \preccurlyeq Y_{j}\right)$ :

$\mathrm{E}\left\{\mathrm{I}\left(Y_{i} \preccurlyeq Y_{j}\right) \mid \mathbf{X}_{i}, \mathbf{X}_{j}\right\}=m\left(\mathbf{X}_{i}, \mathbf{X}_{j} ; \boldsymbol{\beta}_{0}\right), \quad$ with $\mathrm{I}\left(Y_{i} \preccurlyeq Y_{j}\right):=\mathrm{I}\left(Y_{i}<Y_{j}\right)+0.5 \mathrm{I}\left(Y_{i}=Y_{j}\right)$,

where $\mathrm{I}(\cdot)$ denotes the ordinary indicator function so that $\mathrm{I}(A)=1$ if $A$ is true and 0 otherwise. Thas et al. (2012) propose a semiparametric consistent estimator of $\boldsymbol{\beta}_{0}$ by mimicking quasi-likelihood estimating equations with an independence working correlation matrix. As pointed out by several discussants of their paper, this heuristic estimator is not necessarily semiparametrically efficient under correct specification of (1.1) (Van Keilegom, 2012; Leng and Cheng, 2012; Oja, 2012). This is because the pseudoobservations are cross-correlated, e.g., I $\left(Y_{i} \preccurlyeq Y_{j}\right)$ and $\mathrm{I}\left(Y_{i} \preccurlyeq Y_{k}\right)$ are dependent since they share the outcome $Y_{i}$. The potential inefficiency is a consequence of the limitations of the estimation theory of Thas et al. (2012) which only allows for an independence working correlation matrix: the estimation theory ignores the cross-correlation of the pseudo-observations. 
We therefore develop a more general estimation theory. Specifically, we derive the class of all consistent and asymptotically normal estimators of $\boldsymbol{\beta}$ in the semiparametric model induced by (1.1) by appealing to the theory of semiparametrics and we identify the efficient influence function of $\boldsymbol{\beta}$ using on a Hoeffding decomposition (Newey, 1990; Tsiatis, 2006). Next, we propose estimating equations based on the efficient influence function whose solution equals a locally efficient estimator of $\boldsymbol{\beta}$. A semiparametric locally efficient estimator of $\boldsymbol{\beta}$ is obtained by exploiting the relationship between PIMs and semiparametric transformation models Cheng et al. 1995), allowing us to empirically evaluate the efficient estimator under a variety of scenarios. A second estimator is proposed which reduces the second-order bias of the estimator of Thas et al. (2012), where the latter is referred to as the standard estimator. Since the standard, efficient and bias-reduced estimator have a computation complexity of at least $O\left(n^{2}\right)$, we propose computationally more convenient, but asymptotically equivalent variants of these estimators based on partitioning the data. In practice, this partition estimator will especially be useful for the standard estimator.

The article is organised as follows. In Section 2, we present the main results of the estimation theory, identify the efficient influence function and construct a locally efficient estimator. We also discuss the computational 
complexity of these estimators. In Section 3, we study the efficiency and bias properties of this estimator for a variety of well chosen data generating models, while in Section 4 we illustrate the methodology on a case study. In Section 5, the main results of the paper are discussed and we put these results in perspective.

\section{Estimation theory}

We denote the semiparametric model imposed by (1.1) by $\mathcal{M}_{\mathrm{PIM}}$, which is the set of all joint density functions $f_{\mathbf{Z}}(\mathbf{z} ; \boldsymbol{\beta}, \boldsymbol{\eta})=f_{Y \mid \mathbf{X}}\left(y \mid \mathbf{x} ; \boldsymbol{\beta}, \boldsymbol{\eta}_{1}\right) f_{\mathbf{X}}\left(\mathbf{x} ; \boldsymbol{\eta}_{2}\right)$, with $\mathbf{z}^{T}=\left(y, \mathbf{x}^{T}\right)$, obeying 1.1 with $\boldsymbol{\beta}$ the $p$-dimensional parameter of interest and $\boldsymbol{\eta}=\left(\boldsymbol{\eta}_{1}^{T}, \boldsymbol{\eta}_{2}^{T}\right)^{T}$ a (possibly) infinite-dimensional vector of variation independent nuisance parameters. Let the data generating law be $f_{0}(\mathbf{z})=f_{\mathbf{Z}}\left(\mathbf{z} ; \boldsymbol{\beta}_{0}, \boldsymbol{\eta}_{0}\right)=f_{Y \mid \mathbf{X}}\left(y \mid \mathbf{x} ; \boldsymbol{\beta}_{0}, \boldsymbol{\eta}_{10}\right) f_{\mathbf{X}}\left(\mathbf{x} ; \boldsymbol{\eta}_{20}\right)$.

For example, a logistic PIM can be formulated as $\operatorname{logit}\left\{\mathrm{P}\left(Y_{i} \preccurlyeq Y_{j} \mid \mathbf{X}_{i}, \mathbf{X}_{j}\right)\right\}=$ $\left(\mathbf{X}_{j}-\mathbf{X}_{i}\right)^{T} \boldsymbol{\beta}_{0}$, where $\operatorname{logit}(x)=\log \{x /(1-x)\}$. The standard estimator of $\boldsymbol{\beta}_{0}$ in this model solves the estimating equation

$$
\sum_{i=1}^{n} \sum_{j=1}^{n}\left(\mathbf{X}_{j}-\mathbf{X}_{i}\right)\left[\mathrm{I}_{i j}-\operatorname{expit}\left\{\left(\mathbf{X}_{j}-\mathbf{X}_{i}\right)^{T} \boldsymbol{\beta}\right\}\right]=\mathbf{0}
$$

where $\mathrm{I}_{i j}=\mathrm{I}\left(Y_{i} \preccurlyeq Y_{j}\right)$ and $\operatorname{expit}(x)=1 /\left(1+e^{-x}\right)$. Alternatively, a PIM with probit-link can be formulated: $\Phi^{-1}\left\{\mathrm{P}\left(Y_{i} \preccurlyeq Y_{j} \mid \mathbf{X}_{i}, \mathbf{X}_{j}\right)\right\}=\left(\mathbf{X}_{j}-\right.$ $\left.\mathbf{X}_{i}\right)^{T} \boldsymbol{\beta}_{0}$, where $\Phi^{-1}(\cdot)$ is the inverse cumulative standard normal distribu- 
tion function. In this case, the standard estimator of $\boldsymbol{\beta}_{0}$ solves the estimating equation

$$
\sum_{i=1}^{n} \sum_{j=1}^{n}\left(\mathbf{X}_{j}-\mathbf{X}_{i}\right) \frac{\phi\left\{\left(\mathbf{X}_{j}-\mathbf{X}_{i}\right)^{T} \boldsymbol{\beta}\right\}}{\Phi\left\{\left(\mathbf{X}_{j}-\mathbf{X}_{i}\right)^{T} \boldsymbol{\beta}\right\}\left[1-\Phi\left\{\left(\mathbf{X}_{j}-\mathbf{X}_{i}\right)^{T} \boldsymbol{\beta}\right\}\right]}\left[\mathrm{I}_{i j}-\Phi\left\{\left(\mathbf{X}_{j}-\mathbf{X}_{i}\right)^{T} \boldsymbol{\beta}\right\}\right]=\mathbf{0}
$$

and $\phi(\cdot)$ the standard normal density function.

The solutions to $(2.3)$ and $(2.4)$ ignore the cross-correlation of the pseudo-observations. They may hence fail to exploit all available information in the data and can therefore fail to be efficient. To overcome this, we appeal to the theory of semiparametrics to derive the set of all unbiased estimating functions for $\boldsymbol{\beta}$ in model $\mathcal{M}_{\mathrm{PIM}}$ (up to asymptotic equivalence) and to identify the estimating function that leads to the (locally) most efficient estimator.

The set of all unbiased estimating functions for the $p$-dimensional parameter $\boldsymbol{\beta}$ under model $\mathcal{M}_{\mathrm{PIM}}$ is obtained by utilizing the relationship between regular asymptotically linear (RAL) estimators and the geometry of influence functions (Newey, 1990; Tsiatis, 2006). Specifically, an estimator $\widehat{\boldsymbol{\beta}}$ of $\boldsymbol{\beta}$ based on the IID data $\left\{\mathbf{Z}_{i}^{T}=\left(Y_{i}, \mathbf{X}_{i}^{T}\right): i=1, \ldots, n\right\}$ is said to be asymptotically linear under model $\mathcal{M}_{\text {PIM }}$ if it obeys the expansion

$$
\sqrt{n}\left(\widehat{\boldsymbol{\beta}}-\boldsymbol{\beta}_{0}\right)=\frac{1}{\sqrt{n}} \sum_{i=1}^{n} \boldsymbol{\varphi}\left(Y_{i}, \mathbf{X}_{i} ; \boldsymbol{\beta}_{0}, \boldsymbol{\eta}_{0}\right)+o_{p}(1)
$$


for a $p$-dimensional function $\boldsymbol{\varphi}\left(\cdot ; \boldsymbol{\beta}_{0}, \boldsymbol{\eta}_{0}\right)$ of the observed data satisfying the moment conditions $(i) \mathrm{E}\left\{\boldsymbol{\varphi}\left(Y_{i}, \mathbf{X}_{i} ; \boldsymbol{\beta}_{0}, \boldsymbol{\eta}_{0}\right)\right\}=\mathbf{0},(i i) \mathrm{E}\left\{\boldsymbol{\varphi}^{T}\left(Y_{i}, \mathbf{X}_{i} ; \boldsymbol{\beta}_{0}, \boldsymbol{\eta}_{0}\right) \boldsymbol{\varphi}\left(Y_{i}, \mathbf{X}_{i} ; \boldsymbol{\beta}_{0}, \boldsymbol{\eta}_{0}\right)\right\}<$ $\infty$, and $(i i i) \mathrm{E}\left\{\boldsymbol{\varphi}\left(Y_{i}, \mathbf{X}_{i} ; \boldsymbol{\beta}_{0}, \boldsymbol{\eta}_{0}\right) \boldsymbol{\varphi}^{T}\left(Y_{i}, \mathbf{X}_{i} ; \boldsymbol{\beta}_{0}, \boldsymbol{\eta}_{0}\right)\right\}$ is non-singular and where $o_{p}(1)$ denotes a term that converges to zero in probability under the true data generating law $f_{0}(\mathbf{z})$. This $p$-dimensional function $\boldsymbol{\varphi}\left(Y_{i}, \mathbf{X}_{i} ; \boldsymbol{\beta}_{0}, \boldsymbol{\eta}_{0}\right)$ is referred to as the $i$ th influence function $(i=1, \ldots, n)$ of the RAL estimator $\widehat{\boldsymbol{\beta}}$, which is consistent and asymptotically normal with asymptotic variance of $\sqrt{n}\left(\widehat{\boldsymbol{\beta}}-\boldsymbol{\beta}_{0}\right)$ given by $\mathrm{E}\left\{\boldsymbol{\varphi}\left(Y, \mathbf{X} ; \boldsymbol{\beta}_{0}, \boldsymbol{\eta}_{0}\right) \boldsymbol{\varphi}^{T}\left(Y, \mathbf{X} ; \boldsymbol{\beta}_{0}, \boldsymbol{\eta}_{0}\right)\right\}$, the variance of the influence function. We restrict our development to regular estimators, thereby excluding estimators that have undesirable local properties (Newey, 1990) such as super-efficiency. Because the influence function of a RAL estimator $\widehat{\boldsymbol{\beta}}$ is asymptotically uniquely determined, it fully describes the first-order asymptotic behavior of the estimator $\widehat{\boldsymbol{\beta}}$. We therefore focus on identifying the set of all such influence functions from which we can subsequently construct unbiased estimating functions for $\boldsymbol{\beta}_{0}$. Next we identify the influence function with smallest variance, from which a (locally) efficient RAL estimator can be constructed.

Theorem 1 gives the set of all unbiased estimating functions for $\boldsymbol{\beta}_{0}$ in model $\mathcal{M}_{\mathrm{PIM}}$, delivering the class of all RAL estimators of $\boldsymbol{\beta}_{0}$ in model $\mathcal{M}_{\text {PIM }}$ (up to asymptotic equivalence) together with their corresponding 
influence function and asymptotic distribution. The proof of Theorem 1 is given in Section 1 and Section 2 of the online supplementary material.

Theorem 1. If $\widehat{\boldsymbol{\beta}}$ is a RAL estimator of $\boldsymbol{\beta}_{0}$ in model $\mathcal{M}_{\mathrm{PIM}}$, then there exists a p-dimensional function $\mathbf{B}_{i j}(\boldsymbol{\beta})=\mathbf{b}\left(\mathbf{X}_{i}, \mathbf{X}_{j} ; \boldsymbol{\beta}\right)$ in the set $\mathcal{B}$ of antisymmetric p-dimensional functions of $\mathbf{X}_{i}$ and $\mathbf{X}_{j}$ (satisfying $\mathbf{b}\left(\mathbf{X}_{i}, \mathbf{X}_{j} ; \boldsymbol{\beta}\right)+$ $\left.\mathbf{b}\left(\mathbf{X}_{j}, \mathbf{X}_{i} ; \boldsymbol{\beta}\right)=\mathbf{0}\right)$ so that $\widehat{\boldsymbol{\beta}}$ is asymptotically equivalent to the solution of the estimating equation

$$
\sum_{i=1}^{n} \sum_{j=1}^{n} \mathbf{U}_{i j}(\boldsymbol{\beta})=\mathbf{0}, \quad \text { with } \quad \mathbf{U}_{i j}(\boldsymbol{\beta})=\mathbf{B}_{i j}(\boldsymbol{\beta})\left\{\mathrm{I}_{i j}-M_{i j}(\boldsymbol{\beta})\right\},
$$

$\mathrm{I}_{i j}=\mathrm{I}\left(Y_{i} \preccurlyeq Y_{j}\right)$ and $M_{i j}(\boldsymbol{\beta})=m\left(\mathbf{X}_{i}, \mathbf{X}_{j} ; \boldsymbol{\beta}\right)$. Under suitable smoothness and regularity conditions (listed in Section 1 and Section 2 of the online supplementary material), the estimator $\widehat{\boldsymbol{\beta}}$ of $\boldsymbol{\beta}_{0}$ obeys expansion (2.5) with influence function $\boldsymbol{\varphi}\left(Y_{i}, \mathbf{X}_{i} ; \boldsymbol{\beta}_{0}, \boldsymbol{\eta}_{0}\right)=\boldsymbol{C}_{0} \mathrm{E}\left\{\mathbf{U}_{i j}\left(\boldsymbol{\beta}_{0}\right) \mid Y_{i}, \mathbf{X}_{i}\right\}$ and normalization constant $\boldsymbol{C}_{0}=-2 \mathbf{J}\left(\boldsymbol{\beta}_{0}\right)^{-1}$ with $\mathbf{J}\left(\boldsymbol{\beta}_{0}\right)=\mathrm{E}\left\{\partial \mathbf{U}_{i j}(\boldsymbol{\beta}) /\left.\partial \boldsymbol{\beta}^{T}\right|_{\boldsymbol{\beta}=\boldsymbol{\beta}_{0}}\right\}$. It follows that $\sqrt{n}(\widehat{\boldsymbol{\beta}}-\boldsymbol{\beta}) \stackrel{d}{\rightarrow} \mathrm{N}\left(\mathbf{0}, \boldsymbol{\Sigma}_{0}\right)$ with variance-covariance matrix $\boldsymbol{\Sigma}_{0}=4 \mathbf{J}\left(\boldsymbol{\beta}_{0}\right)^{-1} \operatorname{cov}\left[\mathrm{E}\left\{\mathbf{U}_{i j}\left(\boldsymbol{\beta}_{0}\right) \mid Y_{i}, \mathbf{X}_{i}\right\}\right] \mathbf{J}\left(\boldsymbol{\beta}_{0}\right)^{-T}$.

A consistent estimator for the asymptotic variance $\boldsymbol{\Sigma}_{0}$ can be obtained using the sandwich formula: $\widehat{\boldsymbol{\Sigma}}(\widehat{\boldsymbol{\beta}})=4 \widehat{\mathbf{J}}(\widehat{\boldsymbol{\beta}})^{-1} \widehat{\mathbf{K}}(\widehat{\boldsymbol{\beta}}) \widehat{\mathbf{J}}(\widehat{\boldsymbol{\beta}})^{-T}$, with

$$
\widehat{\mathbf{J}}(\widehat{\boldsymbol{\beta}})=\left.\frac{1}{n^{2}} \sum_{i=1}^{n} \sum_{j=1}^{n} \frac{\partial \mathbf{U}_{i j}(\boldsymbol{\beta})}{\partial \boldsymbol{\beta}^{T}}\right|_{\boldsymbol{\beta}=\widehat{\boldsymbol{\beta}}} \text { and } \quad \widehat{\mathbf{K}}(\widehat{\boldsymbol{\beta}})=\frac{1}{n} \sum_{i=1}^{n} \overline{\mathbf{U}}_{i}(\widehat{\boldsymbol{\beta}}) \overline{\mathbf{U}}_{i}^{T}(\widehat{\boldsymbol{\beta}}), \quad \overline{\mathbf{U}}_{i}(\widehat{\boldsymbol{\beta}})=\frac{1}{n} \sum_{j=1}^{n} \mathbf{U}_{i j}(\widehat{\boldsymbol{\beta}})
$$


2.1 The standard estimator of Thas et al. (2012)

\subsection{The standard estimator of Thas et al. (2012)}

We denote the estimator of Thas et al. (2012) as $\widehat{\boldsymbol{\beta}}^{\mathrm{ST}}$ and refer to it as the standard estimator. Before we identify the element of $\mathcal{B}$ that corresponds to the most efficient estimator, we illustrate how the estimating equations proposed in Thas et al. (2012) are a special case of (2.6). In particular, their equations 2.3 and 2.4 are obtained by choosing $\mathbf{B}_{i j}(\boldsymbol{\beta})=$ $\mathbf{B}_{i j}^{\mathrm{ST}}(\boldsymbol{\beta})=\mathbf{b}^{\mathrm{ST}}\left(\mathbf{X}_{i}, \mathbf{X}_{j} ; \boldsymbol{\beta}\right)=\left\{\partial M_{i j}(\boldsymbol{\beta}) / \partial \boldsymbol{\beta}\right\} /\left[M_{i j}(\boldsymbol{\beta})\left\{1-M_{i j}(\boldsymbol{\beta})\right\}\right]$, where the denominator corresponds to the conditional variance of the pseudoobservations $\mathrm{I}_{i j}$ given the covariates $\mathbf{X}_{i}$ and $\mathbf{X}_{j}$, mimicking quasi-likelihood estimating equations with an independence working correlation matrix. For the logistic PIM, 2.3p corresponds to $\mathbf{B}_{i j}^{\mathrm{ST}}(\boldsymbol{\beta})=\left(\mathbf{X}_{j}-\mathbf{X}_{i}\right)$ and for the probit-PIM, 2.4 corresponds to the choice $\mathbf{B}_{i j}^{\mathrm{ST}}(\boldsymbol{\beta})=\left(\mathbf{X}_{j}-\mathbf{X}_{i}\right) \phi\left\{\left(\mathbf{X}_{j}-\right.\right.$ $\left.\left.\mathbf{X}_{i}\right)^{T} \boldsymbol{\beta}\right\}\left(\Phi\left\{\left(\mathbf{X}_{j}-\mathbf{X}_{i}\right)^{T} \boldsymbol{\beta}\right\}\left[1-\Phi\left\{\left(\mathbf{X}_{j}-\mathbf{X}_{i}\right)^{T} \boldsymbol{\beta}\right\}\right]\right)^{-1}$. It can be readily seen that indeed $\mathbf{B}_{i j}^{\mathrm{ST}}(\boldsymbol{\beta})+\mathbf{B}_{j i}^{\mathrm{ST}}(\boldsymbol{\beta})=\mathbf{0}$. Since these estimating equations ignore the cross-correlation structure of the transformed outcomes $\mathrm{I}\left(Y_{i} \preccurlyeq Y_{j}\right)$, they may not deliver an efficient estimator.

\subsection{The locally efficient estimator}

Different choices of the function $\mathbf{B}_{i j}(\boldsymbol{\beta})$ result in RAL estimators with different asymptotic variance. Theorem 2 identifies the choice $\mathbf{B}_{i j}^{\mathrm{EFF}}(\boldsymbol{\beta})=$ 


\subsection{The locally efficient estimator}

$\mathbf{b}^{\mathrm{EFF}}\left(\mathbf{X}_{i}, \mathbf{X}_{j} ; \boldsymbol{\beta}\right)$ that results in the efficient RAL estimator $\widehat{\boldsymbol{\beta}}^{\mathrm{EFF}}$ under model $\mathcal{M}_{\text {PIM }}$. The proof of Theorem 2 is outlined in broad strokes below while a detailed proof is given in Section 3 of the online supplementary material.

Theorem 2. The efficient estimator $\widehat{\boldsymbol{\beta}}^{\mathrm{EFF}}$ is obtained by choosing $\mathbf{B}_{i j}(\boldsymbol{\beta})=$ $\mathbf{B}_{i j}^{\mathrm{EFF}}(\boldsymbol{\beta})$ in $(2.6)$, where $\mathbf{B}_{i j}^{\mathrm{EFF}}(\boldsymbol{\beta})$ is the solution to the integral equation

$\mathbf{D}_{i j}\left(\boldsymbol{\beta}_{0}\right)=\mathrm{E}\left\{\mathbf{B}_{i k}^{\mathrm{EFF}}\left(\boldsymbol{\beta}_{0}\right) V_{i j i k}\left(\boldsymbol{\beta}_{0}\right)+\mathbf{B}_{j k}^{\mathrm{EFF}}\left(\boldsymbol{\beta}_{0}\right) V_{i j j k}\left(\boldsymbol{\beta}_{0}\right) \mid \mathbf{X}_{i}, \mathbf{X}_{j}\right\}, \quad i \neq k$ and $j \neq k$,

with $\mathbf{D}_{i j}\left(\boldsymbol{\beta}_{0}\right)=\partial M_{i j}(\boldsymbol{\beta}) /\left.\partial \boldsymbol{\beta}\right|_{\boldsymbol{\beta}=\boldsymbol{\beta}_{0}}$ and with conditional covariance

$$
V_{i j k l}\left(\boldsymbol{\beta}_{0}\right)=V\left(\mathbf{X}_{i}, \mathbf{X}_{j}, \mathbf{X}_{k}, \mathbf{X}_{l} ; \boldsymbol{\beta}_{0}\right)=\operatorname{cov}\left(\mathrm{I}_{i j}, \mathrm{I}_{k l} \mid \mathbf{X}_{i}, \mathbf{X}_{j}, \mathbf{X}_{k}, \mathbf{X}_{l}\right)
$$

Proof. The semiparametric nuisance tangent space $\Lambda$ of model $\mathcal{M}_{\text {PIM }}$ equals

$$
\Lambda=\left\{\mathbf{s}(Y, \mathbf{X}) \in \mathcal{H} \mid \mathrm{E}\left[\left\{\mathbf{s}(Y, \mathbf{X})+\mathbf{s}\left(Y^{*}, \mathbf{X}^{*}\right)\right\}\left\{\mathrm{I}\left(Y \preccurlyeq Y^{*}\right)-m\left(\mathbf{X}, \mathbf{X}^{*} ; \boldsymbol{\beta}_{0}\right)\right\} \mid \mathbf{X}, \mathbf{X}^{*}\right]=\mathbf{0}\right\}
$$

for $\left(Y, \mathbf{X}^{T}\right) \Perp\left(Y^{*}, \mathbf{X}^{*, T}\right)$ and $\mathcal{H}$ the Hilbert space equipped with the covariance inner product of $p$-dimensional mean-zero and square integrable measurable random functions $\mathbf{h}(Y, \mathbf{X})$. The orthogonal complement of the 


\subsection{The locally efficient estimator}

semiparametric nuisance tangent space $\Lambda^{\perp}$ equals

$$
\begin{array}{r}
\Lambda^{\perp}=\left\{\mathbf{s}^{\perp}(Y, \mathbf{X}) \in \mathcal{H} \mid \mathbf{s}^{\perp}(Y, \mathbf{X})=\mathrm{E}\left[\mathbf{b}\left(\mathbf{X}, \mathbf{X}^{*}\right)\left\{\mathrm{I}\left(Y \preccurlyeq Y^{*}\right)-m\left(\mathbf{X}, \mathbf{X}^{*} ; \boldsymbol{\beta}_{0}\right)\right\} \mid Y, \mathbf{X}\right],(2.9)\right. \\
\left.\mathbf{b}\left(\mathbf{X}, \mathbf{X}^{*}\right) \in \mathcal{B}\right\},
\end{array}
$$

for $\left(Y, \mathbf{X}^{T}\right) \Perp\left(Y^{*}, \mathbf{X}^{*, T}\right)$, where $\mathcal{B}=\left\{\mathbf{b}\left(\mathbf{X}, \mathbf{X}^{*}\right) \mid \mathbf{b}\left(\mathbf{X}, \mathbf{X}^{*}\right)\right.$ is square integrable and $\mathbf{b}\left(\mathbf{X}, \mathbf{X}^{*}\right)+$ $\left.\mathbf{b}\left(\mathbf{X}^{*}, \mathbf{X}\right)=\mathbf{0}\right\}$. The score function for $\boldsymbol{\beta}$ equals $\mathbf{s}_{\boldsymbol{\beta}}\left(Y, \mathbf{X} ; \boldsymbol{\beta}_{0}, \boldsymbol{\eta}_{0}\right)=\partial \log f_{Y \mathbf{X}}\left(y, \mathbf{x} ; \boldsymbol{\beta}, \boldsymbol{\eta}_{0}\right) /\left.\partial \boldsymbol{\beta}\right|_{\boldsymbol{\beta}=\boldsymbol{\beta}_{0}}$ and the efficient influence function is given by

$\boldsymbol{\varphi}^{\mathrm{EFF}}\left(Y, \mathbf{X} ; \boldsymbol{\beta}_{0}, \boldsymbol{\eta}\right)=\mathrm{E}\left\{\mathbf{s}^{\mathrm{EFF}}\left(Y, \mathbf{X} ; \boldsymbol{\beta}_{0}, \boldsymbol{\eta}_{0}\right) \mathbf{s}^{\mathrm{EFF}, T}\left(Y, \mathbf{X} ; \boldsymbol{\beta}_{0}, \boldsymbol{\eta}_{0}\right)\right\}^{-1} \mathbf{s}^{\mathrm{EFF}}\left(Y, \mathbf{X} ; \boldsymbol{\beta}_{0}, \boldsymbol{\eta}_{0}\right)$,

with efficient score $\mathbf{s}^{\mathrm{EFF}}\left(Y, \mathbf{X} ; \boldsymbol{\beta}_{0}, \boldsymbol{\eta}_{0}\right)$ equal to the orthogonal projection of the score function onto the complement of the nuisance tangent space. In order to find the efficient score, we thus need to find the function $\mathbf{b}^{\mathrm{EFF}}\left(\mathbf{X}, \mathbf{X}^{*}\right) \in \mathcal{B}$ so that $\mathbf{s}^{\mathrm{EFF}}\left(Y, \mathbf{X} ; \boldsymbol{\beta}_{0}, \boldsymbol{\eta}_{0}\right)=\mathrm{E}\left[\mathbf{b}^{\mathrm{EFF}}\left(\mathbf{X}, \mathbf{X}^{*}\right)\left\{\mathrm{I}\left(Y \preccurlyeq Y^{*}\right)-\right.\right.$ $\left.\left.m\left(\mathbf{X}, \mathbf{X}^{*} ; \boldsymbol{\beta}_{0}\right)\right\} \mid Y, \mathbf{X}\right]$. This boils down to solving the integral equation

$$
\begin{aligned}
& \mathrm{E}\left[\left\{\mathbf{s}_{\boldsymbol{\beta}}\left(Y, \mathbf{X} ; \boldsymbol{\beta}_{0}, \boldsymbol{\eta}_{0}\right)+\mathbf{s}_{\boldsymbol{\beta}}\left(Y^{*}, \mathbf{X}^{*} ; \boldsymbol{\beta}_{0}, \boldsymbol{\eta}_{0}\right)\right\}\left\{\mathrm{I}\left(Y \preccurlyeq Y^{*}\right)-m\left(\mathbf{X}, \mathbf{X}^{*} ; \boldsymbol{\beta}_{0}\right)\right\} \mid \mathbf{X}, \mathbf{X}^{*}\right] \\
& =\mathrm{E}\left(\left[\mathbf{b}^{\mathrm{EFF}}\left(\mathbf{X}, \mathbf{X}^{\dagger}\right)\left\{\mathrm{I}\left(Y \preccurlyeq Y^{\dagger}\right)-m\left(\mathbf{X}, \mathbf{X}^{\dagger} ; \boldsymbol{\beta}_{0}\right)\right\}+\mathbf{b}^{\mathrm{EFF}}\left(\mathbf{X}^{*}, \mathbf{X}^{\dagger}\right)\left\{\mathrm{I}\left(Y^{*} \preccurlyeq Y^{\dagger}\right)-m\left(\mathbf{X}^{*}, \mathbf{X}^{\dagger} ; \boldsymbol{\beta}_{0}\right)\right\}\right]\right. \\
& \left.\quad \times\left\{\mathrm{I}\left(Y \preccurlyeq Y^{*}\right)-m\left(\mathbf{X}, \mathbf{X}^{*} ; \boldsymbol{\beta}_{0}\right)\right\} \mid \mathbf{X}, \mathbf{X}^{*}\right),
\end{aligned}
$$

from which expression 2.7$)$ follows because $\mathrm{E}\left[\left\{\mathbf{s}_{\boldsymbol{\beta}}^{T}\left(Y, \mathbf{X} ; \boldsymbol{\beta}_{0}, \boldsymbol{\eta}_{0}\right)+\mathbf{s}_{\boldsymbol{\beta}}^{T}\left(Y^{*}, \mathbf{X}^{*} ; \boldsymbol{\beta}_{0}, \boldsymbol{\eta}_{0}\right)\right\}\{\mathrm{I}(Y \preccurlyeq\right.$ $\left.\left.\left.Y^{*}\right)-m\left(\mathbf{X}, \mathbf{X}^{*} ; \boldsymbol{\beta}_{0}\right)\right\} \mid \mathbf{X}, \mathbf{X}^{*}\right]=\partial m\left(\mathbf{X}, \mathbf{X}^{*} ; \boldsymbol{\beta}\right) /\left.\partial \boldsymbol{\beta}^{T}\right|_{\boldsymbol{\beta}=\boldsymbol{\beta}_{0}}$ for $\mathcal{M}_{\mathrm{PIM}}$. 
2.2 The locally efficient estimator

Unfortunately, the integral equation (2.7) does not admit a closed form solution for the function $\mathbf{B}_{i j}^{\mathrm{EFF}}(\boldsymbol{\beta})$, especially when the conditional covariances $V_{i j k l}(\boldsymbol{\beta})$ depend on $\boldsymbol{\beta}$. It therefore needs to be solved numerically using computationally demanding iterative procedures. In doing so, we replace the expectation in (2.7) by its empirical counterpart, where the average is taken over $k=1, \ldots, n$. Specifically, for a fixed $\boldsymbol{\beta}$, we can approximate $\mathbf{B}_{i j}^{\mathrm{EFF}}(\boldsymbol{\beta})$ by $\widehat{\mathbf{B}}_{i j}^{\mathrm{EFF}}(\boldsymbol{\beta})$ where $\widehat{\mathbf{B}}_{i j}^{\mathrm{EFF}}(\boldsymbol{\beta})$ solves the linear system of equations

$\mathbf{D}_{i j}(\boldsymbol{\beta})=\frac{1}{n} \sum_{k=1}^{n}\left\{\widehat{\mathbf{B}}_{i k}^{\mathrm{EFF}}(\boldsymbol{\beta}) V_{i j i k}(\boldsymbol{\beta})+\widehat{\mathbf{B}}_{j k}^{\mathrm{EFF}}(\boldsymbol{\beta}) V_{i j j k}(\boldsymbol{\beta})\right\}, \quad i, j=1, \ldots, n$.

From their defining properties, it easily follows that for arbitrary $k, \ell \in$ $\{1, \ldots, n\}$ we have that $\mathbf{D}_{k \ell}(\boldsymbol{\beta})=-\mathbf{D}_{\ell k}(\boldsymbol{\beta}), \widehat{\mathbf{B}}_{k \ell}^{\mathrm{EFF}}(\boldsymbol{\beta})=-\widehat{\mathbf{B}}_{\ell k}^{\mathrm{EFF}}(\boldsymbol{\beta})$ and $V_{i j k \ell}(\boldsymbol{\beta})=-V_{i j \ell k}(\boldsymbol{\beta})=V_{k \ell i j}(\boldsymbol{\beta})$. In particular, this implies that for all $i \in\{1, \ldots, n\}, \mathbf{D}_{i i}(\boldsymbol{\beta})=\mathbf{0}, \mathbf{B}_{i i}^{\mathrm{EFF}}(\boldsymbol{\beta})=\widehat{\mathbf{B}}_{i i}^{\mathrm{EFF}}(\boldsymbol{\beta})=\mathbf{0}$ and $V_{i i k \ell}(\boldsymbol{\beta})=0$.

We can conclude that the equations for which $i=j$ do not contribute to the system. The antisymmetry conditions additionally guarantee that the equation $\mathbf{D}_{i j}(\boldsymbol{\beta})=n^{-1} \sum_{k=1}^{n}\left\{\widehat{\mathbf{B}}_{i k}^{\mathrm{EFF}}(\boldsymbol{\beta}) V_{i j i k}(\boldsymbol{\beta})+\widehat{\mathbf{B}}_{j k}^{\mathrm{EFF}}(\boldsymbol{\beta}) V_{i j j k}(\boldsymbol{\beta})\right\}$ is equivalent to the equation

$$
\mathbf{D}_{j i}(\boldsymbol{\beta})=\frac{1}{n} \sum_{k=1}^{n}\left\{\widehat{\mathbf{B}}_{j k}^{\mathrm{EFF}}(\boldsymbol{\beta}) V_{j i j k}(\boldsymbol{\beta})+\widehat{\mathbf{B}}_{i k}^{\mathrm{EFF}}(\boldsymbol{\beta}) V_{j i i k}(\boldsymbol{\beta})\right\}
$$


2.2 The locally efficient estimator

and the linear system of equations consequently reduces to those $n(n-1) / 2$ equations with $i<j$. When $\{i, j\} \cap\{k, \ell\}=\emptyset$, the pseudo-observations $\mathrm{I}_{i j}$ and $\mathrm{I}_{k \ell}$ are uncorrelated so that $V_{i j k \ell}(\boldsymbol{\beta})=0$. Using this, we conclude that the linear system of $n^{2}$ equations 2.10 is equivalent to the linear system of $n(n-1) / 2$ equations

$\mathbf{D}_{i j}(\boldsymbol{\beta})=\frac{1}{n}\left[\sum_{k=1}^{n-1} \sum_{\ell=k+1}^{n}\left\{\widehat{\mathbf{B}}_{k \ell}^{\mathrm{EFF}}(\boldsymbol{\beta}) V_{i j k \ell}(\boldsymbol{\beta})\right\}+\widehat{\mathbf{B}}_{i j}^{\mathrm{EFF}}(\boldsymbol{\beta}) V_{i j i j}(\boldsymbol{\beta})\right], \quad i<j$

A detailed calculation of this last step is provided in the online supplementary material (Section 4$)$. Now define the $[n(n-1) / 2 \times p]$-dimensional matrices $\mathbf{D}(\boldsymbol{\beta})$ and $\widehat{\mathbf{B}}^{\mathrm{EFF}}(\boldsymbol{\beta})$ so that the $[(i-1)(2 n-i) / 2+j-i]$-th row corresponds to $\mathbf{D}_{i j}^{T}(\boldsymbol{\beta})$ and $\widehat{\mathbf{B}}_{i j}^{\mathrm{EFF}, T}(\boldsymbol{\beta})$, respectively. Next, define the $[n(n-1) / 2 \times n(n-1) / 2]$-dimensional matrix $\mathbf{V}(\boldsymbol{\beta})$ so that $V_{i j k \ell}(\boldsymbol{\beta})$ is on the $[(i-1)(2 n-i) / 2+j-i]$-th row and $[(k-1)(2 n-k) / 2+\ell-k]$-th column Finally, define the $[n(n-1) / 2 \times n(n-1) / 2]$-dimensional diagonal matrix $\mathbf{V}_{\text {indep }}(\boldsymbol{\beta})$ so that the $[(i-1)(2 n-i) / 2+j-i]$-th diagonal element equals $V_{i j i j}(\boldsymbol{\beta})$. In this notation, 2.11 can be written as the matrix equation $n \mathbf{D}(\boldsymbol{\beta})=\widehat{\mathbf{B}}^{\mathrm{EFF}, T}(\boldsymbol{\beta})\left\{\mathbf{V}(\boldsymbol{\beta})+\mathbf{V}_{\text {indep }}(\boldsymbol{\beta})\right\}$. It follows that

$$
\widehat{\mathbf{B}}^{\mathrm{EFF}}(\boldsymbol{\beta})=n \mathbf{D}^{T}(\boldsymbol{\beta})\left\{\mathbf{V}(\boldsymbol{\beta})+\mathbf{V}_{\text {indep }}(\boldsymbol{\beta})\right\}^{-1}
$$

A semiparametric efficient estimator $\widehat{\boldsymbol{\beta}}^{\mathrm{EFF}}$ can then be obtained by itera- 


\subsection{The locally efficient estimator}

tively solving the estimating equation $\widehat{\mathbf{B}}^{\mathrm{EFF}, T}(\boldsymbol{\beta})\{\mathbf{I}-\mathbf{M}(\boldsymbol{\beta})\}=\mathbf{0}$, with $\mathbf{I}$ and $\mathbf{M}(\boldsymbol{\beta})$ both $n(n-1) / 2$-dimensional vectors so that the $[(i-1)(2 n-$ $i) / 2+j-i]$-th element is given by $\mathrm{I}_{i j}$ and $M_{i j}(\boldsymbol{\beta})$, respectively.

Remark 1. Theorem 1 does not cover the setting where $\widehat{\mathbf{B}}(\boldsymbol{\beta})$ is replaced by an estimator. However, substituting $\widehat{\mathbf{B}}(\boldsymbol{\beta})$ by an estimator does not affect the asymptotic distribution of the resulting estimator of $\boldsymbol{\beta}$ because by construction the considered estimator's influence function is orthogonal to the nuisance parameter space Tsiatis (2006). This implies that its asymptotic behavior is the same whether it is evaluated at the true nuisance parameters versus estimated nuisance parameters that converge at a faster than $n^{1 / 4}$ rate to the truth.

Remark 2. Let $\mathbf{B}^{\mathrm{ST}}(\boldsymbol{\beta})$ denote the $[n(n-1) / 2 \times p]$-dimensional matrix with $[(i-1)(2 n-i) / 2+j-i]$-th row equal to $\mathbf{B}_{i j}^{\mathrm{ST}, T}(\boldsymbol{\beta})$. Using the above notation, we have that $\mathbf{B}^{\mathrm{ST}}(\boldsymbol{\beta})=\mathbf{D}^{T}(\boldsymbol{\beta}) \mathbf{V}_{\text {indep }}^{-1}(\boldsymbol{\beta})$. It follows that $\widehat{\boldsymbol{\beta}}^{\mathrm{ST}}$ solves the estimating equation $\mathbf{D}^{T}(\boldsymbol{\beta}) \mathbf{V}_{\text {indep }}^{-1}(\boldsymbol{\beta})\{\mathbf{I}-\mathbf{M}(\boldsymbol{\beta})\}=\mathbf{0}$. That this estimator ignores the cross-correlation between the pseudo-observations is clearly visualized here because $\mathbf{B}^{\mathrm{ST}}(\boldsymbol{\beta})$ can be obtained from $\widehat{\mathbf{B}}^{\mathrm{EFF}}(\boldsymbol{\beta})$ by enforcing $\mathbf{V}(\boldsymbol{\beta})$ to be zero.

Remark 2 illustrates how the complex cross-correlation structure of the 
2.2 The locally efficient estimator

pseudo-observations is taken into account by the semiparametric efficient estimator $\widehat{\boldsymbol{\beta}}^{\mathrm{EFF}}$. It is the $[n(n-1) / 2 \times n(n-1) / 2]$-dimensional matrix $\mathbf{V}(\boldsymbol{\beta})$ in equation 2.12 , consisting of the elements $V_{i j k l}(\boldsymbol{\beta})$ (the correlations between the pseudo-observations) that shows how the information contained within these correlation coefficients is exploited by the semiparametric efficient estimator.

We still need to address one peculiarity. When solving the integral equation (2.7) numerically, we need reasonable estimators for the covariances $V_{i j k l}(\boldsymbol{\beta})$ since nonparametric estimation is unstable or even unfeasible in small samples due to the curse of dimensionality Robins and Ritov, 1997). We therefore need to impose an additional working model, as we will illustrate for a continuous outcome. In this case, the resulting estimator $\widehat{\boldsymbol{\beta}}^{\mathrm{EFF}}$ will only be locally efficient and not globally efficient. That is, the semiparametric efficiency bound is only attained under a correctly specified working model for the covariance structure but not necessarily otherwise. Under misspecification of the working model, the consistency of the estimator is maintained.

When the outcome $Y$ is continuous, in which case the conditional probabilistic index satisfies $\mathrm{P}\left(Y_{i} \preccurlyeq Y_{j} \mid \mathbf{X}_{i}, \mathbf{X}_{j}\right)=\mathrm{P}\left(Y_{i}<Y_{j} \mid \mathbf{X}_{i}, \mathbf{X}_{j}\right)$, the con- 


\subsection{The locally efficient estimator}

ditional covariance can be written as

$$
V_{i j i k}(\boldsymbol{\beta})=\mathrm{P}\left(Y_{i}<\min \left(Y_{j}, Y_{k}\right) \mid \mathbf{X}_{i}, \mathbf{X}_{j}, \mathbf{X}_{k}\right)-M_{i j}(\boldsymbol{\beta}) M_{i k}(\boldsymbol{\beta})
$$

and

$$
V_{i j j k}(\boldsymbol{\beta})=\mathrm{P}\left(Y_{i}<Y_{j}<Y_{k} \mid \mathbf{X}_{i}, \mathbf{X}_{j}, \mathbf{X}_{k}\right)-M_{i j}(\boldsymbol{\beta}) M_{j k}(\boldsymbol{\beta})
$$

Consequently, to compute the efficient estimator, one needs to model probabilities of the form $\mathrm{P}\left(Y_{i}<\min \left(Y_{j}, Y_{k}\right) \mid \mathbf{X}_{i}, \mathbf{X}_{j}, \mathbf{X}_{k}\right)$ and $\mathrm{P}\left(Y_{i}<Y_{j}<Y_{k} \mid \mathbf{X}_{i}, \mathbf{X}_{j}, \mathbf{X}_{k}\right)$. One flexible way of doing this, is by assuming a semiparametric transformation model (STM)

$$
H(Y)=\mathbf{X}^{T} \boldsymbol{\alpha}+\varepsilon
$$

where $\varepsilon$ is a zero-mean random error term with a known cumulative distribution function $F_{\varepsilon}(\cdot)$ and $H(\cdot)$ is an unspecified strictly increasing function (e.g. Cuzick, 1988; Cheng et al., 1995). Under such model, it follows that

$$
\mathrm{P}\left(Y_{i}<Y_{j} \mid \mathbf{X}_{i}, \mathbf{X}_{j}\right)=f^{-1}\left\{\left(\mathbf{X}_{j}-\mathbf{X}_{i}\right)^{T} \boldsymbol{\alpha}\right\}
$$

with $f^{-1}(a)=\int F_{\varepsilon}(a+b) d F_{\varepsilon}(b)$. For example, if $\varepsilon$ is standard normally distributed, $F_{\varepsilon}(a)=\Phi(a)$ and from this it follows that $f^{-1}(a)=\Phi(a / \sqrt{2})$, resulting in a probit-PIM with $\boldsymbol{\beta}=\boldsymbol{\alpha} / \sqrt{2}$. Alternatively, if $\varepsilon$ follows the Gumbel distribution (with location parameter 0 and scale parameter 1), $F_{\varepsilon}(a)=\exp \{-\exp (-a)\}$, then $f^{-1}(a)=\operatorname{expit}(a)$, resulting in a logistic PIM with $\boldsymbol{\beta}=\boldsymbol{\alpha}$. Table 1 summarizes these relationships. 


\subsection{A bias-reduced estimator}

Table 1: Relationship between the semiparametric transformation model and the probabilistic index model for two choices of error distribution $F_{\varepsilon}$.

Semiparametric Transformation Model Probabilistic Index Model

$$
H\left(Y_{i}\right)=\mathbf{X}_{i}^{T} \boldsymbol{\alpha}+\varepsilon_{i} \quad \mathrm{P}\left(Y_{i}<Y_{j} \mid \mathbf{X}_{i}, \mathbf{X}_{j}\right)=g^{-1}\left\{\left(\mathbf{X}_{j}-\mathbf{X}_{i}\right)^{T} \boldsymbol{\beta}\right\}
$$

Normal error $F_{\varepsilon}(a)=\Phi(a)$

$g^{-1}(a)=\Phi(a)$ and $\boldsymbol{\beta}=\boldsymbol{\alpha} / \sqrt{2}$

Gumbel error $F_{\varepsilon}(a)=\exp \{-\exp (-a)\}$

$$
g^{-1}(a)=\operatorname{expit}(a) \text { and } \boldsymbol{\beta}=\boldsymbol{\alpha}
$$

One can further show for the semiparametric transformation model that

$$
\begin{aligned}
& \mathrm{P}\left(Y_{i}<\min \left(Y_{j}, Y_{k}\right) \mid \mathbf{X}_{i}, \mathbf{X}_{j}, \mathbf{X}_{k}\right) \\
= & h_{1}^{-1}\left\{\left(\mathbf{X}_{i}-\mathbf{X}_{j}\right)^{T} \boldsymbol{\alpha},\left(\mathbf{X}_{i}-\mathbf{X}_{k}\right)^{T} \boldsymbol{\alpha}\right\}, \\
& \mathrm{P}\left(Y_{i}<Y_{j}<Y_{k} \mid \mathbf{X}_{i}, \mathbf{X}_{j}, \mathbf{X}_{k}\right) \\
= & h_{2}^{-1}\left\{\left(\mathbf{X}_{j}-\mathbf{X}_{i}\right)^{T} \boldsymbol{\alpha},\left(\mathbf{X}_{j}-\mathbf{X}_{k}\right)^{T} \boldsymbol{\alpha}\right\},
\end{aligned}
$$

with $h_{1}^{-1}(a, b)=\int\left\{1-F_{\varepsilon}(a+c)\right\}\left\{1-F_{\varepsilon}(b+c)\right\} d F_{\varepsilon}(c)$ and $h_{2}^{-1}(a, b)=$ $\int F_{\varepsilon}(a+c)\left\{1-F_{\varepsilon}(b+c)\right\} d F_{\varepsilon}(c)$. The functions $h_{1}^{-1}(\cdot, \cdot)$ and $h_{2}^{-1}(\cdot, \cdot)$ can be obtained via numerical integration.

\subsection{A bias-reduced estimator}

Obtaining the locally efficient estimator $\widehat{\boldsymbol{\beta}}^{\mathrm{EFF}}$ is computationally expensive, especially when the covariances $V_{i j k l}(\boldsymbol{\beta})$ depend on the parameter $\boldsymbol{\beta}$. We 
2.3 A bias-reduced estimator

here propose a simplification that comes with some computational advantage. Instead of using $V_{i j k l}(\boldsymbol{\beta})$ where $\boldsymbol{\beta}$ is treated as a running parameter, we will fix the value of $\boldsymbol{\beta}$ to a prespecified value $\boldsymbol{\beta}^{*}$. This results in the covariances $V_{i j k l}\left(\boldsymbol{\beta}^{*}\right)$ and the estimating equation $\sum_{i=1}^{n} \sum_{j=1}^{n} \mathbf{U}_{i j}^{*}(\boldsymbol{\beta})=\mathbf{0}$, with $\mathbf{U}_{i j}^{*}(\boldsymbol{\beta})=\widehat{\mathbf{B}}_{i j}^{*}(\boldsymbol{\beta})\left\{\mathrm{I}_{i j}-M_{i j}(\boldsymbol{\beta})\right\}$ in which $\widehat{\mathbf{B}}_{i j}^{*}(\boldsymbol{\beta})$ solves

$$
\mathbf{D}_{i j}(\boldsymbol{\beta})=\frac{1}{n} \sum_{k=1}^{n}\left\{\widehat{\mathbf{B}}_{i k}^{*}(\boldsymbol{\beta}) V_{i j i k}\left(\boldsymbol{\beta}^{*}\right)+\widehat{\mathbf{B}}_{j k}^{*}(\boldsymbol{\beta}) V_{i j j k}\left(\boldsymbol{\beta}^{*}\right)\right\},
$$

delivering $\widehat{\mathbf{B}}^{*}(\boldsymbol{\beta})=n \mathbf{D}^{T}(\boldsymbol{\beta})\left\{\mathbf{V}\left(\boldsymbol{\beta}^{*}\right)+\mathbf{V}_{\text {indep }}\left(\boldsymbol{\beta}^{*}\right)\right\}^{-1}$. The quantity $\widehat{\mathbf{B}}_{i j}^{*}(\boldsymbol{\beta})$ serves as the numerical approximation of $\mathbf{B}_{i j}^{*}(\boldsymbol{\beta})$, the solution to the integral equation (2.7) including the aforementioned simplification. This procedure requires the inversion of the (model-based) variance-covariance matrix of the pseudo-observations only once. This is in contrast to the calculation of the locally efficient estimator which requires inversion of this variancecovariance matrix in every step of the iterative procedure. This results in a computational gain.

The solution to $\sum_{i=1}^{n} \sum_{j=1}^{n} \mathbf{U}_{i j}^{*}(\boldsymbol{\beta})=\mathbf{0}$ gives the estimator $\widehat{\boldsymbol{\beta}}^{*}$. Because this estimation procedure constitutes a special case of 2.6) (for any choice of $\left.\boldsymbol{\beta}^{*}\right)$, where the function $\mathbf{B}_{i j}(\boldsymbol{\beta})$ is set to $\widehat{\mathbf{B}}_{i j}^{*}(\boldsymbol{\beta})$, the estimator $\widehat{\boldsymbol{\beta}}^{*}$ is a consistent and asymptotically normal estimator of $\boldsymbol{\beta}$.

An important question that remains is how to choose the value $\boldsymbol{\beta}^{*}$. This value was selected in order to minimize the second-order finite-sample 
2.3 A bias-reduced estimator

bias of $\hat{\boldsymbol{\beta}}^{*}$. This is addressed in the following Proposition 1. A proof and regularity conditions are given in the online supplementary material (Section 5).

Proposition 1. Assume that the semiparametric transformation model (2.13) holds and let $\varepsilon$ follow a symmetric distribution about zero. Then, under regularity conditions, the second-order finite-sample bias of $\widehat{\boldsymbol{\beta}}^{*}$ is minimized at $\boldsymbol{\beta}^{*}=\mathbf{0}$.

When we set $\boldsymbol{\beta}^{*}=\mathbf{0}$, we denote the estimator $\widehat{\boldsymbol{\beta}}^{*}$ by $\widehat{\boldsymbol{\beta}}^{\mathrm{BR}}$, the biasreduced estimator (this explains the superscript $\mathrm{BR}$ ). When the true value $\boldsymbol{\beta}_{0}$ equals $\mathbf{0}$, the covariances $V_{i j k l}(\boldsymbol{\beta})$ are correctly modeled given correct specification of the STM. In this case (under the null), the estimator $\widehat{\boldsymbol{\beta}}^{\mathrm{BR}}$ is also semiparametric efficient under model $\mathcal{M}_{\mathrm{PIM}}$.

Remark 3. The working model 2.13 is parameterized by the nuisance parameter $\boldsymbol{\alpha}$, which is a function of the parameter of interest $\boldsymbol{\beta}$; say $\boldsymbol{\alpha}=\mathbf{k}(\boldsymbol{\beta})$. For the locally efficient estimator, $\boldsymbol{\alpha}$ is treated as a running parameter, while for the biased-reduced estimator it is set to $\boldsymbol{\alpha}=\mathbf{0}$. Other estimators for $\boldsymbol{\beta}$ might be constructed by estimating $\boldsymbol{\alpha}$ in 2.13 directly, e.g. using rank likelihood (Cuzick, 1988), and treat it as fixed while solving 2.6 for $\boldsymbol{\beta}$. 
2.4 Computational issues and solutions

\subsection{Computational issues and solutions}

Before empirically studying the theoretical properties of the different estimators, we first focus on their computational properties. All three estimators requiring solving a system of equations of the form (2.6). The computational difference between the estimators lies in the computation of $\mathbf{B}_{i j}(\boldsymbol{\beta})$. Equation 2.12 gives its expression for $\widehat{\boldsymbol{\beta}}^{\mathrm{EFF}}$ : it involves inverting a matrix of dimension $n(n-1) / 2 \times n(n-1) / 2$. When an iterative algorithm (e.g. a Newton method) is used to solve (2.6), this matrix has to be inverted at each iteration. The estimator $\widehat{\boldsymbol{\beta}}^{\mathrm{BR}}$ is computationally less intensive as it only needs the inversion once and not at each iteration as $\mathbf{B}_{i j}\left(\boldsymbol{\beta}^{*}\right)$ is not a function of $\boldsymbol{\beta}$ but is instead kept fixed at $\boldsymbol{\beta}^{*}=\mathbf{0}$. The estimator $\widehat{\boldsymbol{\beta}}^{\mathrm{ST}}$ is the computationally least demanding of all three as it does not require the inversion of an $n(n-1) / 2 \times n(n-1) / 2$ matrix because $\mathbf{B}_{i j}(\boldsymbol{\beta})$ has a simple expression of the form $\mathbf{B}_{i j}(\boldsymbol{\beta})=\left\{\partial M_{i j}(\boldsymbol{\beta}) / \partial \boldsymbol{\beta}\right\} /\left[M_{i j}(\boldsymbol{\beta})\left\{1-M_{i j}(\boldsymbol{\beta})\right\}\right]$.

We refer to Section 6 of the online supplementary material for computation times of the three estimators for several sample sizes.

Despite its computational superiority, $\widehat{\boldsymbol{\beta}}^{\mathrm{ST}}$ is still computationally demanding because of the double summation in (2.6) which can be problematic for large $n$. We therefore propose a partition estimator which is computationally less demanding, but asymptotically equivalent. 
2.4 Computational issues and solutions

For this purpose, we partition the data in $k$ distinct parts $S_{i}(i=$ $1, \ldots, k)$ of size $\left|S_{i}\right|=m_{i}$ so that $\sum_{i=1}^{k} m_{i}=n, m_{i} \rightarrow \infty$ as $n \rightarrow \infty$ and $k \rightarrow \infty$ and $k / \sqrt{n} \rightarrow 0$ as $n \rightarrow \infty$. Let $\widehat{\boldsymbol{\beta}}_{i}$ denote any RAL estimator applied to part $i$ of the data. The partition estimator is then given by

$$
\widetilde{\boldsymbol{\beta}}:=\frac{1}{n} \sum_{i=1}^{k} m_{i} \cdot \widehat{\boldsymbol{\beta}}_{i}
$$

Theorem 3 shows first-order asymptotic equivalence of the partition estimator $\widetilde{\boldsymbol{\beta}}$ and the corresponding RAL estimator $\widehat{\boldsymbol{\beta}}$ applied to the entire dataset without partitioning.

Theorem 3. Under model $\mathcal{M}_{\mathrm{PIM}}$, the partition estimator $\widetilde{\boldsymbol{\beta}}=n^{-1} \sum_{i=1}^{k} m_{i}$. $\widehat{\boldsymbol{\beta}}_{i}\left(\widehat{\boldsymbol{\beta}}_{i}\right.$ a consistent estimator applied to part $i$ of the data, $\left.i=1, \ldots, k\right)$ is a consistent estimator for $\boldsymbol{\beta}_{0}$. It further holds that $\sqrt{n}\left(\widetilde{\boldsymbol{\beta}}-\boldsymbol{\beta}_{0}\right)=$ $\sqrt{n}\left(\widehat{\boldsymbol{\beta}}-\boldsymbol{\beta}_{0}\right)+o_{p}(1)$, with $\widehat{\boldsymbol{\beta}}$ the estimator applied to the entire dataset without partitioning.

A proof of Theorem 3 is given in Section 7 of the the online supplementary material. It thus follows from Theorem 3 that $\sqrt{n}\left(\widetilde{\boldsymbol{\beta}}-\boldsymbol{\beta}_{0}\right)$ and $\sqrt{n}\left(\widehat{\boldsymbol{\beta}}-\boldsymbol{\beta}_{0}\right)$ have the same limiting distribution. A consistent estimator of the variance of $\widetilde{\boldsymbol{\beta}}$ is obtained from

$$
\operatorname{Var}(\widetilde{\boldsymbol{\beta}})=\frac{1}{n^{2}} \sum_{i=1}^{k} m_{i}^{2} \cdot \operatorname{Var}\left(\widehat{\boldsymbol{\beta}}_{i}\right)
$$


with $\operatorname{Var}\left(\widehat{\boldsymbol{\beta}}_{i}\right)$ replaced by the sandwich estimator from Theorem 1 .

In practice we propose $k=\left\lfloor n^{0.5-\delta}\right\rfloor$ with $0<\delta<0.5$ and $\lfloor x\rfloor$ the integer part of $x$, and to partition the data in $k$ groups such that $\max _{i=1, \ldots, k}\left(m_{i}\right)-$ $\min _{i=1, \ldots, k}\left(m_{i}\right) \leq 1$. The partition estimator can then be substantially faster since it requires $k$ estimates on a subset of size $m_{i}$ instead of one estimate on the entire dataset of size $n$. In practice, $\delta$ might be chosen so that computational complexity is minimized.

\section{Empirical evaluation}

To study the empirical performance of the estimators of Section 2, data are generated under the linear transformation model 2.13). The strictly increasing function $H(\cdot)$ is set to the identity function. Recall that Table 1 summarizes the relationship between the data-generating model and the PIM.

All simulations are done in $\mathrm{R}$ ( $\mathrm{R}$ Core Team, 2018) and all R-code can be downloaded from goo.gl/UA4mFV.

\subsection{Normally distributed error}

We start by considering a normally distributed error in model (2.13), which corresponds to a probit-PIM. We consider a univariate covariate $X$ that fol- 
3.1 Normally distributed error

lows a discrete uniform distribution with $K$ support values equally spaced between $(a, u)$. For the following simulation experiments, we set $a$ to 0.1 , $K$ to 10 and evaluate different values of the upper limit $u$. By using a discrete covariate, equation (2.7) reduces to a summation that, following steps 2.10)- 2.12), allows a closed-form solution for $\mathbf{B}^{\mathrm{EFF}}(\boldsymbol{\beta})$. In this way, we avoid having to approximate equation (2.7) so that differences in efficiency, if any, are solely due to the estimators themselves. We can empirically study the range of potential efficiency gain by considering specific simulation scenarios where small and larger gains in efficiency of $\widehat{\boldsymbol{\beta}}^{\mathrm{EFF}}$ over $\widehat{\boldsymbol{\beta}}^{\mathrm{ST}}$ are theoretically expected. We do this by selecting values of the data-generating model so that the difference (in terms of the Frobenius norm) between the estimating equations of $\widehat{\boldsymbol{\beta}}^{\mathrm{EFF}}$ and $\widehat{\boldsymbol{\beta}}^{\mathrm{ST}}$ is maximized (leading to a scenario where we expect $\widehat{\boldsymbol{\beta}}^{\mathrm{EFF}}$ to perform better) or minimized (leading to a scenario where we expect a similar performance of both estimators). We refer to the online supplementary material (Section 8) for more information on the rationale on how to obtain these simulation scenarios.

Specifically, we consider $u=2$ and $\alpha=0$ (leading to a scenario where the efficient estimator is expected to perform better than $\widehat{\boldsymbol{\beta}}^{\mathrm{ST}}$ ) or $\alpha=2$ (leading to a scenario where $\widehat{\boldsymbol{\beta}}^{\mathrm{EFF}}$ is expected to have a similar performance than $\widehat{\boldsymbol{\beta}}^{\mathrm{ST}}$ ) corresponding to true values $\beta_{0}=0$ or $\beta_{0}=\sqrt{2}$ respectively. The 
3.1 Normally distributed error

simulation results can be found in Section 8 of the online supplementary material. In what follows we describe the main findings.

The simulation results indicate that $\widehat{\boldsymbol{\beta}}^{\mathrm{ST}}$ is nearly as efficient as $\widehat{\boldsymbol{\beta}}^{\mathrm{EFF}}$ for both choices of $\beta_{0}$, suggesting that the information that is lost by not considering the cross-correlation of the pseudo-observations is negligible, especially when the sample size increases. This is interesting, especially from a computational point of view: $\widehat{\boldsymbol{\beta}}^{\mathrm{ST}}$ is computationally substantially less intensive than the semiparametric efficient estimator $\widehat{\boldsymbol{\beta}}^{\mathrm{EFF}}$ since the latter requires calculating and inverting a covariance matrix of dimension $n(n-1) / 2$. This behavior can be intuitively explained as follows. The number of non-zero elements of $\left\{\mathbf{V}(\boldsymbol{\beta})+\mathbf{V}_{\text {indep }}(\boldsymbol{\beta})\right\}$ in expression 2.12 equals $n(n-1)(n-3 / 2)$. The sparsity of this matrix equals $(4 n-6) /[n(n-1)]$ which converges to zero with increasing sample size. Hence, with increasing sample size, the variance-covariance matrix of the pseudo-observations becomes sparser, with most of the significant information on the diagonal. This supports the use of the standard estimator in larger samples instead of the locally efficient or even the bias-reduced estimator, in which case finite-sample bias becomes a lesser concern.

The simulation results also demonstrate the local efficiency property of $\widehat{\boldsymbol{\beta}}^{\mathrm{BR}}$. Its relative efficiency, compared to $\widehat{\boldsymbol{\beta}}^{\mathrm{EFF}}$, is close to 1 when $\beta_{0}=0$, 


\subsection{Gumbel distributed error}

but increases, i.e., $\widehat{\boldsymbol{\beta}}^{\mathrm{BR}}$ becomes less efficient than $\widehat{\boldsymbol{\beta}}^{\mathrm{EFF}}$, when the true $\beta_{0}$ deviates from zero. This is because the covariance structure of $\widehat{\boldsymbol{\beta}}^{\mathrm{BR}}$ is no longer correctly specified when $\beta_{0} \neq 0$, leading to inefficient but still consistent estimates. Its bias reduction property is also noticeable, especially when $\beta_{0}=\sqrt{2}$, at small sample sizes. This bias reduction comes at a price of higher standard errors.

For small sample sizes, especially for $n=25$, the empirical variance is underestimated by the sandwich estimator of the standard error (for all estimators). In these small sample settings, one could use resampling techniques such as the adjusted jackknife empirical likelihood method (as detailed in Amorim et al. (2018)) to obtain appropriate confidence intervals with correct coverage. The coverage here using the asymptotic results is better for $n=100$.

\subsection{Gumbel distributed error}

We now consider the case where the error term $\varepsilon$ in model 2.13 follows a Gumbel distribution with location parameter zero and scale parameter 1, which leads to a logistic PIM; see Table 1. Similar as before, we consider specific simulation scenarios where small and larger gains in efficiency are expected of $\widehat{\boldsymbol{\beta}}^{\mathrm{EFF}}$ over $\widehat{\boldsymbol{\beta}}^{\mathrm{ST}}$. The rationale behind these settings is discussed 
3.2 Gumbel distributed error

in the online supplementary material (Section 8). Because the Gumbel distribution of the error is not symmetric around zero, this scenario also allows us to investigate to what extent the result of Proposition 1 is retained here.

To better understand the restrictions imposed by probabilistic index models, we also add $\widehat{\boldsymbol{\beta}}^{\mathrm{PH}}$ (the Cox partial likelihood estimator) to the simulation study. A semiparametric transformation model with Gumbel error is equivalent to the Cox proportional hazard model, so that $\widehat{\boldsymbol{\beta}}^{\mathrm{PH}}$ is the efficient estimator under this more restrictive semiparametric transformation model. The simulation results can be found in Section 8 of the online supplemental material. We discuss the main findings below.

The estimator $\widehat{\boldsymbol{\beta}}^{\mathrm{PH}}$ is more efficient than all competitors; it is around $20 \%$ more efficient than $\widehat{\boldsymbol{\beta}}^{\mathrm{EFF}}$, regardless of the sample size. This is because proportional hazard models, or more generally, semiparametric transformation models, are more restrictive than PIMs. In the online supplementary material (Section 9), we argue in more detail why this is the case and that this is not in contradiction to the semiparametric theory developed in this paper. The relationship between these two approaches is further discussed in Section 5 .

With respect to the three PIM estimators (those that are within the 


\subsection{Partition estimator}

class of RAL estimators given in Theorem 1), we notice that for $\beta_{0}=0$, there is almost no gain in efficiency and all three estimators show similar performance. When $\beta_{0}=2$ and for a sample of size 25 , both $\widehat{\boldsymbol{\beta}}^{\mathrm{BR}}$ and $\widehat{\boldsymbol{\beta}}^{\mathrm{EFF}}$ have a lower mean squared error (MSE) as compared to $\widehat{\boldsymbol{\beta}}^{\mathrm{ST}}$. Notice that the covariance structure used in the construction of $\widehat{\boldsymbol{\beta}}^{\mathrm{BR}}$ is misspecified when $\beta_{0}=2$, but its smaller bias contributes to a smaller MSE. Also notice that, even though the assumptions of Proposition 1 are not fulfilled (the Gumbel distribution is not symmetric around zero), $\widehat{\boldsymbol{\beta}}^{\mathrm{BR}}$ shows smaller bias than the other estimators, contributing to a lower MSE when the sample size is small.

As the sample size increases, all three estimators are nearly unbiased and the estimator $\widehat{\boldsymbol{\beta}}^{\mathrm{ST}}$ shows the largest MSE. The efficient estimator $\widehat{\boldsymbol{\beta}}^{\mathrm{EFF}}$ performs better than both competitors, although the improvement is modest. This gain in efficiency however comes with a considerable cost in computing time. Finally, the empirical coverage of $95 \%$ CIs is again similar as before.

\subsection{Partition estimator}

We also examine the finite sample performance of the partition estimator $\widetilde{\boldsymbol{\beta}}$ of Section 2.4. We only consider the standard estimator. This is because the 


\subsection{Conclusion}

partition estimator is only of interest for large sample sizes and we want to compare $\widetilde{\boldsymbol{\beta}}$ with $\widehat{\boldsymbol{\beta}}$. Furthermore, calculation of $\widehat{\boldsymbol{\beta}}^{\mathrm{BR}}$ and $\widehat{\boldsymbol{\beta}}^{\mathrm{EFF}}$ (nonpartitioned) also becomes infeasible for large sample sizes. Section 8 of the online supplemental material shows the empirical results of the partition estimator. For $n \geq 500$ the partition estimator $\widetilde{\boldsymbol{\beta}}^{\mathrm{ST}}$ is almost as efficient as $\widehat{\boldsymbol{\beta}}^{\text {ST }}$. The partition variance estimator exhibits a slight underestimation, but this reduces with increasing sample size. Overall we can say that the distributions of $\widetilde{\boldsymbol{\beta}}^{\mathrm{ST}}$ and $\widehat{\boldsymbol{\beta}}^{\mathrm{ST}}$ are approximately equal for $n \geq 500$.

\subsection{Conclusion}

The results of this empirical evaluation suggest that in many settings there is practically no difference in efficiency between $\widehat{\boldsymbol{\beta}}^{\mathrm{ST}}$ and both $\widehat{\boldsymbol{\beta}}^{\mathrm{EFF}}$ and $\widehat{\boldsymbol{\beta}}^{\mathrm{BR}}$, and when there is a difference, its magnitude is modest. Therefore $\widehat{\boldsymbol{\beta}}^{\mathrm{ST}}$ be considered the preferred estimator in practice given its computational superiority over the other estimators.

\section{Illustration}

The Health Evaluation and Linkage to Primary Care study is a clinical trial for adult inpatients recruited from a detoxification unit. The data are made available in Appendix B of Horton and Kleinman (2010). To illustrate how 
PIMs can supplement conventional analyses, we consider a cross-sectional part of the original study with $n=453$ and focus on the association between substance abuse consequence and depression symptoms while controlling for gender (1: female, 0: male) and homelessness (1: homeless at least one night in the last six months, 0 : otherwise). The analyses are done in $R(\bar{R}$ Core Team, 2018) and all R-code for this analysis can be downloaded from goo.gl/UA4mFV.

Substance abuse consequence is the primary outcome and is measured by the Inventory of Drug Use Consequences (InDUC, range 0-50) based on 50 items where higher scores indicate worse life consequences (Blanchard et al., 2001). Since the InDUC score is an ordinal outcome, the probabilistic index is a relevant summary measure. Depression is measured by the Center for Epidemiologic Studies Depression Scale (CESD, range 0-60) with higher scores indicating more symptoms of depression. To visualize the association, Figure 1 (left panel) shows a scatter plot of the InDUC score as a function of CESD together with the fit of a linear regression model controlling for gender and homeless status. To allow for some flexibility CESD is modeled as a third order polynomial. There is a positive association between the depression score and the mean substance abuse consequence score. The plot further demonstrates a decreasing outcome variability with increasing 
CESD score. Transforming the outcome according to a Box-Cox power function stabilizes the variance and makes the residuals approximately normal; see the online supplementary material for details (Section 10). The data generating model can therefore be approximated with a probit-link PIM. We refer to Section 2.2 for more details on the connection between a transformation model and a PIM.

For all estimators we consider the partition estimator of Section 2.4 with $k=\left\lfloor n^{0.25}\right\rfloor=\left\lfloor 453^{0.25}\right\rfloor=4$ partitions, while $\widehat{\boldsymbol{\beta}}^{\mathrm{ST}}$ is also applied to the dataset without partitioning.

Table 2: Estimates and standard errors of the three estimators when data are partitioned for computational reasons. The two columns on the right show the estimates and the standard errors when fitted to the dataset without partitioning.

\begin{tabular}{|c|c|c|c|c|c|c|c|c|}
\hline & \multirow[b]{2}{*}{$\widetilde{\boldsymbol{\beta}}^{\mathrm{ST}}$} & \multicolumn{3}{|c|}{ With partitioning } & \multirow[b]{2}{*}{$\widetilde{\boldsymbol{\beta}}^{\mathrm{EFF}}$} & \multirow[b]{2}{*}{ St. Error } & \multicolumn{2}{|c|}{ Without partitioning } \\
\hline & & St. Error & $\widetilde{\boldsymbol{\beta}}^{\mathrm{BR}}$ & St. Error & & & $\widehat{\boldsymbol{\beta}}^{\mathrm{ST}}$ & St. Error \\
\hline CESD & 0.09708 & 0.03155 & 0.09657 & 0.03284 & 0.10037 & 0.03220 & 0.07704 & 0.03735 \\
\hline $\mathrm{CESD}^{2}$ & -0.00249 & 0.00112 & -0.00232 & 0.00119 & -0.00260 & 0.00117 & -0.00197 & 0.00127 \\
\hline $\mathrm{CESD}^{3}$ & 0.00003 & 0.00001 & 0.00002 & 0.00001 & 0.00003 & 0.00001 & 0.00002 & 0.00001 \\
\hline homeless & 0.34356 & 0.06994 & 0.33512 & 0.07621 & 0.33742 & 0.07658 & 0.32386 & 0.06951 \\
\hline gender & -0.59627 & 0.08633 & -0.59858 & 0.09279 & -0.59578 & 0.09255 & -0.61094 & 0.08684 \\
\hline
\end{tabular}


Table 2 displays the results. All estimates are quite similar, which is in line with the findings of the simulation study. Further note that there is only a small difference with the estimates obtained by fitting the standard estimator on the full dataset. This demonstrates the strength of this estimator: it is computationally superior and nearly efficient. The partition standard errors are smaller than those of the estimator applied to the dataset without partitioning, but this is consequence of the variance estimator underestimation as discussed in Section 3.3.

The regression coefficients of CESD, gender and homeless status are significantly different from zero $(p<0.0001)$. There is no evidence that the association between CESD and the InDUC score (while controlling for gender and homeless status) significantly deviates from linearity $(p=0.22)$.

To illustrate the interpretation, consider two patients of the same gender and with the same homeless status but with a difference of 10 on the depression scale. The probability that the person with the lowest CESD score will have a lower InDUC score is displayed in Figure 1 (right panel) as a function of the lowest CESD score. From this panel we see that lower depression scores are associated with lower substance abuse consequence scores, since the PI is above 0.5. For illustration, consider a patient with a score of 25 and a patient with a score of 35 . The probability that the 

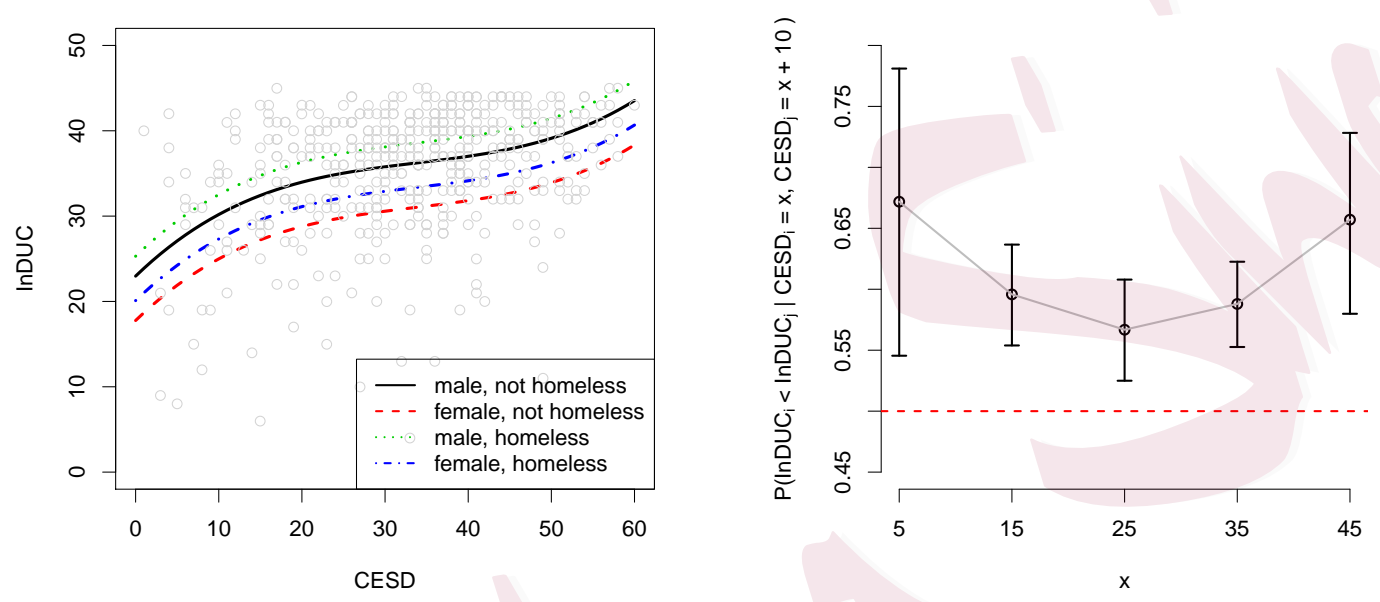

Figure 1: Left: scatter plot of the InDUC score as a function of CESD together with the fit of a linear regression model controlling for gender and homeless status. Right: estimated probability that the InDUC score is lower for a patient with a CESD of $x$ as compared to a patient with a CESD that is 10 units higher (while both patients have the same gender and homelessness status) as a function of $x$. The vertical lines denote the pointwise $95 \%$ confidence intervals. 
patient with the lowest CESD score will also have the lowest InDUC score is

$\Phi\left[0.07704 \cdot(35-25)-0.00197 \cdot\left(35^{2}-25^{2}\right)+0.00002 \cdot\left(35^{3}-25^{3}\right)\right]=56.7 \%$,

where the percentage is calculated using the unrounded values of $\widehat{\boldsymbol{\beta}}^{\text {ST }}$. It is hence more likely that having fewer symptoms of depression is associated with less substance abuse consequences. The effect is however modest, since the probability is close to $50 \%$. From the plot we also see that this effect is not linear: the impact of a 10 units difference in CESD scores on the InDUC scores depends on the CESD scores. For low and high CESD scores, the estimated PI is the largest although less precise.

We want to emphasize the fact that when interpreting the PI, we are comparing two different subpopulations. In the former example, we are comparing two populations of the same gender and with the same homeless status but with a difference of 10 points on the depression scale and with potentially different values for all other (possibly unmeasured) variables. Depending on how heterogeneous these populations are, the PI will be closer to 0.5 when both subpopulations are more heterogeneous and will deviate more from 0.5 when both subpopulations are more homogeneous. The PI is not indicating how much a specific patient would benefit/hurt from a 10 points increase on the depression scale. Likewise, in a 
randomised design, the PI refers to the interpretation of the probability that a randomly selected treated subject has a higher outcome than an independently randomly selected untreated subject. This should not misleadingly be interpreted as the probability to benefit from the treatment. For a more detailed discussion, see for example Senn (2006); Greenland et al. (2019).

\section{Discussion}

We have worked out solid semiparametric theory for PIMs and derived the (locally) efficient estimator $\widehat{\boldsymbol{\beta}}^{\mathrm{EFF}}$ of the parameter $\boldsymbol{\beta}$ indexing these models, where efficiency is attained under additional correct specification of a STM. We proposed a second estimator, $\widehat{\boldsymbol{\beta}}^{\mathrm{BR}}$, with has a local efficiency property and reduced second-order finite-sample bias.

Our results have shown that the standard estimator is nearly efficient under several data-generating mechanisms. This is surprising, considering the correlation between the pseudo-observations, but can be explained by the sparsity of their covariance matrix. This degree of sparsity increases with the sample size $n$. More intuition into this behavior is given in Section 11 of the online supplementary material. In view of this, and its computational efficiency, we recommend the use of the standard estimator. We have further extended the standard estimator by providing a computation- 
ally improved partition estimation strategy. Techniques for sparse matrices will likely result in even more improved computational properties and will be explored in future research.

PIMs might also be used to analyze composite outcomes. Pocock et al. (2012) proposed the win-ratio, which is related to the probabilistic index, as a meaningful effect size. Recently, several authors have proposed methods for modelling the win-ratio as a function of covariates (e.g. Follmann et al. 2020; Mao and Wang, 2021). The estimators of the model parameters are related to the PIM parameter estimators of Thas et al. (2012). However, since composite endpoints often involve time-to-event outcomes (e.g. survival times), censoring is an important issue that has been studied for the estimation of the win-ratio. These insights may perhaps be transferred to the PIMs so that the estimation theory can be extended to censoring. Also the approach of Cheng et al. (1995) in the context of transformation models for censored data, which relies on inverse probability of censoring weighting may be considered.

\section{Supplementary Materials}

This file contains the development of the semiparametric efficiency theory, more detailed proofs and calculations needed in the article, more explana- 


\section{REFERENCES}

tions concerning the simulation set-up, the tables of the simulation study and additional figures for the data analysis. (PDF-file).

\section{Acknowledgements}

The authors would like to thank the Flemish Research Council (FWO) for financial support (Grant G.0202.14N) and the editor, associate editor and the referees for their insightful and constructive comments.

\section{References}

Amorim, G., O. Thas, K. Vermeulen, S. Vansteelandt, and J. De Neve (2018). Small sample inference for probabilistic index models. Computational Statistics and Data Analysis 121, $137-148$

Blanchard, K., J. Morgenstern, T. Morgan, and E. Labourie (2001). Consequences of substance use: psychometric properties of the Inventory of Drug Use Consequences (InDUC). Alcohol and Clinical Experimentation and Research 25(5 supplement), 136A.

Chamberlain, G. (1987). Asymptotic efficiency in estimation with conditional moment restrictions. Journal of Econometrics 34, 305-334.

Cheng, S., L. Wei, and Z. Ying (1995). Analysis of transformation models with censored data. Biometrika 82(4), 835-845.

Cuzick, J. (1988). Rank regression. Annals of Statistics 16, 1369-1389. 


\section{REFERENCES}

De Neve, J. and O. Thas (2015). A regression framework for rank tests based on the probabilistic index model. Journal of the American Statistical Association 110(511), 1276-1283.

Follmann, D., M. P. Fay, T. Hamasaki, and S. Evans (2020). Analysis of ordered composite endpoints. Statistics in Medicine 39(5), 602-616.

Greenland, S., M. P. Fay, E. H. Brittain, J. H. Shih, D. A. Follmann, E. E. Gabriel, and J. M. Robins (2019). On causal inferences for personalized medicine: How hidden causal assumptions led to erroneous causal claims about the d-value. The American Statistician 0(0), $1-13$.

Horton, N. J. and K. Kleinman (2010). Using $R$ for data management, statistical analysis, and graphics. CRC Press.

Leng, C. and G. Cheng (2012). Discussion of "Probabilistic Index Models" by O.Thas, J. De Neve, L. Clement and J.P. Ottoy. Journal of the Royal Statistical Society - Series B 74, $623-671$.

Mao, L. and T. Wang (2021). A class of proportional win-fractions regression models for composite outcomes. Biometrics $n / a(\mathrm{n} / \mathrm{a})$.

Newey, W. (1988). Adaptive estimation of regression models via moment restrictions. Journal of Econometrics 38, 301-339.

Newey, W. (1990). Semiparametric efficiency bounds. Journal of Applied Econometrics 5(2), $99-135$. 


\section{REFERENCES}

Oja, H. (2012). Discussion of "Probabilistic Index Models" by O.Thas, J. De Neve, L. Clement and J.P. Ottoy. Journal of the Royal Statistical Society - Series B 74, 623-671.

Pocock, S. J., C. A. Ariti, T. J. Collier, and D. Wang (2012). The win ratio: a new approach to the analysis of composite endpoints in clinical trials based on clinical priorities. European Heart Journal 33(2), 176-182.

R Core Team (2018). R: A Language and Environment for Statistical Computing. Vienna, Austria: R Foundation for Statistical Computing.

Robins, J. M. and Y. Ritov (1997). Toward a curse of dimensionality appropriate (CODA) asymptotic theory for semi-parametric models. Statistics in Medicine 16, 285-319.

Senn, S. (2006). Letter to the Editor. Probabilistic index: an intuitive non-parametric approach to measuring the size of the treatment effects by L. Acion, J.J. Peterson, S. Temple and S. Arndt, Statistics in Medicine 2006; 25 (4): 591-602. Statistics in Medicine 25(22), 3944-3946.

Thas, O., J. De Neve, L. Clement, and Ottoy, J.P. (2012). Probabilistic index models (with discussion). Journal of the Royal Statistical Society - Series B 74, 623-671.

Tsiatis, A. A. (2006). Semiparametric Theory and Missing Data. Springer: New York.

Van Keilegom, I. (2012). Discussion of "Probabilistic Index Models" by O.Thas, J. De Neve, L. Clement and J.P. Ottoy. Journal of the Royal Statistical Society - Series B 74, 623-671.

Vermeulen, K., O. Thas, and S. Vansteelandt (2015). Increasing the power of the Mann- 


\section{REFERENCES}

Whitney test in randomized experiments through flexible covariate adjustment. Statistics in Medicine 34(6), 1012-1030.

Department of Data Analysis and Mathematical Modelling, Ghent University, Ghent, Belgium

E-mail: Karelb.Vermeulen@UGent.be

Department of Data Analysis, Ghent University, Ghent, Belgium

E-mail: Jan.DeNeve@UGent.be

Department of Data Analysis and Mathematical Modelling, Ghent University, Ghent, Belgium and Department of Biostatistics, Vanderbilt University Medical Center, Nashville, US.

E-mail: ggca@outlook.com

Department of Data Analysis and Mathematical Modelling, Ghent University, Ghent, Belgium and Center for Statistics, Hasselt University, Hasselt, Belgium and National Institute for Applied Statistics Research Australia (NIASRA), School of Mathematics and Applied Statistics, University of Wollongong, Wollongong, Australia

E-mail: Olivier.Thas@UGent.be

Department of Applied Mathematics, Computer Sciences and Statistics, Ghent University,

Ghent, Belgium and Department of Medical Statistics, London School of Hygiene and Tropical

Medicine, London, United Kingdom

E-mail: Stijn.Vansteelandt@UGent.be 